\title{
DRG aktuell
}

$\mathrm{D}$ ie sporadischen Kurzinformationen $\mathrm{zu}$ aktuellen Reizthemen, Fragen der Kodierung und Auswirkungen auf die Tätigkeit des Krankenhausarztes in unserer Sparte ,DRG aktuell“ finden bei den Lesern große Aufmerksamkeit.

Bekanntlich bietet der Umgang mit den DRGs in den Kliniken weiterhin viel Konfliktstoff. Dies ist umso bedauerlicher, als die wirtschaftliche Situation der Kliniken in hohem Maße davon abhängt, dass dieses System gut beherrscht wird. Als „Anbieter“ der Kurzinformationen kann man naturgemäß nur von selbst wahrgenommenen Problemen ausgehen. Diese mögen nicht immer die gleichen sein, die im Klinikalltag auftauchen. Wir würden aber auch gern dort helfen, wo tatsächlich der Schuh drückt.

Um mit der Sparte „DRG aktuell“ wirklich praktische Hilfe leisten zu können, haben wir deshalb beschlossen, das bisher ausschließlich angebotsorientierte System um ein nachfrageorientiertes System zu erweitern, mit anderen Worten einen Fragekasten zu DR G-Problemen einzurichten. Willkommen sind neben direkt die Kodie- rung betreffenden Fragen auch Hinweise auf typische Fallstricke, häufige Konflikte zwischen Krankenhaus und Kostenträger und Kommentare zu aktuellen Entwicklungen, die einer breiten Leserschaft nähergebracht werden sollen. Es ist erwähnenswert, dass die Publikation der Krankenhausperspektive sich in früheren Fällen als gutes Instrument zur Untermauerung der eigenen Argumentation bewährt hat. Unser neu eingerichteter Fragekasten gibt Ihnen ab jetzt die Möglichkeit, dieses Angebot aktiv zu nutzen!

Der Spartenherausgeber unserer Zeitschrift wird sich bemühen, die eingegangenen Fragen, die ja meist auch von allgemeinerem Interesse sein werden, kurzfristig in seiner Rubrik zu beantworten. Die Fragen sollen in der Regel mit Namensnennung in der Zeitschrift abgedruckt werden. Um möglichen Hemmnissen vorzubeugen, soll aber auch die Möglichkeit bestehen, die Fragen anonym einzusenden. Sie erreichen die Rubrik „DR G aktuell“ über die E-Mail-Adresse drg@ medizinischeklinik.de.

Justus de Zeeuw, Haan 